Revue interdisciplinaire sur la Grèce archaïque

\title{
Quelques réflexions en guise de conclusion
}

A Few Words by Way of Conclusion

\section{Maria Teresa Schettino}

\section{(2) OpenEdition}

Journals

Édition électronique

URL : https://journals.openedition.org/gaia/597

DOI : $10.4000 /$ gaia. 597

ISSN : 2275-4776

\section{Éditeur}

UGA Éditions/Université Grenoble Alpes

\section{Édition imprimée}

ISBN : 978-2-37747-199-7

ISSN : $1287-3349$

\section{Référence électronique}

Maria Teresa Schettino, "Quelques réflexions en guise de conclusion », Gaia [En ligne], 22-23 | 2020, mis en ligne le 01 juin 2020, consulté le 09 décembre 2021. URL : http://journals.openedition.org/gaia/ 597 ; DOI : https://doi.org/10.4000/gaia.597

Ce document a été généré automatiquement le 9 décembre 2021.

Gaia. Revue interdisciplinaire sur la Grèce archaïque 


\title{
Quelques réflexions en guise de conclusion
}

\author{
A Few Words by Way of Conclusion
}

Maria Teresa Schettino

«Cette sorte d'histoire était toute locale. Elle commençait à la fondation, parce que ce qui était antérieur à cette date n'intéressait en rien la cité ; et c'est pourquoi les anciens ont si complètement ignoré leurs origines. Elle ne rapportait aussi que les événements dans lesquels la cité s'était trouvée engagée, et elle ne s'occupait pas du reste

de la terre. Chaque cité avait son histoire spéciale, comme elle avait sa religion et son calendrier. "

(Fustel de Coulanges, La cité antique, Paris 1864,

p. 216)

1 Les mythes et les rites de fondation étaient l'expression de l'identité d'une cité qui remontait au début de son histoire. Aujourd'hui, les traces culturelles et institutionnelles qui se reflètent dans les récits des origines mythiques peuvent être mises à l'épreuve des découvertes archéologiques et des études sur le phénomène de l'urbanisation dans le pourtour méditerranéen. La nouvelle approche a renouvelé la question de l'origine et de la nature de la cité et a permis de mettre au jour des établissements et des agglomérations relevant d'une expérience « urbaine » pour des périodes ou des régions à propos desquelles l'idée même d'« urbanisation » n'était pas acquise dans l'historiographie traditionnelle.

2 C'est dans le cadre de ce renouveau qu'il faut replacer le présent dossier, qui réunit les actes de deux journées d'études organisées par Arianna Esposito et Airton Pollini, à l'université de Dijon, en 2018. Le thème relève d'un des domaines de recherche des deux responsables du dossier, et est au cœur des projets scientifiques qu'ils 
coordonnent dans leurs UMR d'appartenance, dans un esprit de collaboration mutuelle et de synergie fructueuse ${ }^{1}$.

3 L'approche proposée est à la fois analytique et typologique et vise à appréhender le phénomène de l'urbanisme qui marqua la Méditerranée à travers l'étude de la documentation historique, littéraire et archéologique. Le dossier se divise en deux parties. La première est consacrée aux premières formes d'organisation d'une cité-État, de la Crète (Daniela Lefèvre-Novaro) aux sites préurbains de l'Italie antique (Francesco Quondam), des poleis grecques, notamment Sparte (Valeria Tosti) et Naupacte (Despina Chatzivasiliou), aux colonies en Occident, comme Béziers (Élian Gomez et Daniela Ugolini), et en Orient, en particulier en Carie (Gabrièle Larguinat-Turbatte), du monde étrusque (Claire Joncheray) à l'Europe celtique (Andrea Fochesato). La seconde partie complète le cadre proposé dans la section précédente par quatre études lexicales consacrées à autant de régions soumises par Rome: deux concernent les vexatae quaestiones des termes oppidum, à la lumière du Bellum civile de César (Sabine Lefebvre), et castellum dans le contexte de l'Afrique du Nord (Anne-Florence Baroni); la troisième porte sur la Lusitanie au Haut-Empire et le vocabulaire utilisé pour identifier et affirmer une communauté (Susana Marcos); la dernière sur les termes civitas et urbs, notamment dans le contexte de la péninsule Ibérique pendant l'Antiquité tardive (Christian Stein).

4 La naissance de la cité n'est pas un sujet nouveau : en 2004, Mogens Herman Hansen, avec Thomas Heine Nielsen, a publié un monumental inventaire des cités grecques ${ }^{2}$, à la suite de ses célèbres études sur la polis ${ }^{3}$. Ce mot grec fait partie de ceux dont l'interprétation et la traduction divisent depuis longtemps les historiens de l'Antiquité : l'ouvrage Polis and City-State, traduit en français en 2001, part d'un problème de traduction pour mener une analyse comparée de la polis classique des $v^{e}$ et $I^{e}$ siècles av. J.-C. et de l'État moderne du $\mathrm{Xx}^{\mathrm{e}}$ siècle ${ }^{4}$.

5 À quel moment la cité grecque est-elle apparue ? Quand a-t-elle disparu ? Quel rapport entretenait-elle avec son territoire ? Comment était composé le corps civique ? Ce sont certaines des interrogations posées par le livre de Mogens H. Hansen, dans le sillage de nombreuses études précédentes autour de la cité antique. Les réponses apportées dépendent naturellement d'une définition de cette forme de vie en société qui ne caractérisa pas seulement le monde grec antique mais toute la Méditerranée, de l'Occident au Proche-Orient.

6 Si l'approche dans Polis and City-State est surtout diachronique et centrée sur l'évolution de la cité grecque et les colonies, notamment au Proche-Orient, avec une comparaison avec l'État moderne, Mogens H. Hansen propose un regard géographique plus large dans l'ouvrage collectif qu'il a dirigé en 2000, A Comparative Study of 30 City-State Cultures $^{5}$, où l'approche est à la fois synchronique et diachronique, mais ce n'est pas le moment où la cité naît dans différents contextes historiques qui est au centre de ce volume, plutôt la cité dans sa forme complète et organisée.

7 Les études du présent recueil sont en revanche centrées sur les origines du phénomène urbain et le processus qui conduisit à la fondation de cités dans les régions méditerranéennes, et pas seulement dans la sphère politico-culturelle du monde grechellénistique. Ce sujet s'insère dans la longue tradition de travaux aussi bien autour des origines de la cité, que d'études comparatistes dans le cadre de la Méditerranée antique. Notamment, dans le sillage du volume dirigé par Emanuele Greco, La città greca antica. Istituzioni, società e forme urbane (Rome, 1999), les actes réunis ici traitent 
ensemble les deux aspects principaux concernant la cité antique, qui sont souvent demeurés séparés dans la vaste bibliographie sur le sujet jusqu'à la fin des années 1990, à savoir la cité en tant que communauté et la cité dans sa dimension matérielle (comme l'urbs et l'oppidum latins). La longue séparation de ces deux aspects, le premier faisant l'objet d'études historiques et l'autre concernant sa dimension matérielle et archéologique, découle de plusieurs facteurs. Tout d'abord, le point de vue historique a été longtemps et de façon presque exclusive celui de l'histoire politique. Ensuite, les études archéologiques et d'urbanistique n'ont donné un apport important au débat sur la polis en général qu'à partir du xxI siècle. Comme nous l'avons déjà souligné, c'est depuis les années 1990 que la perspective s'est élargie grâce aux recherches menées au Copenhagen Polis Center, dirigé par Mogens H. Hansen, qui se sont imposées comme références car elles tentent la synthèse entre la tradition littéraire, la documentation épigraphique et l'état des connaissances archéologiques ${ }^{6}$. La notion de polis est le résultat de l'union des deux principaux éléments qui la composent, à savoir l'asty (l'espace urbain) et la chora (la campagne).

En ce qui concerne l'aube des villes antiques, comme le dit le titre du dossier, trois de ses articles traitent des changements qui se sont produits à l'occasion de l'effondrement du système palatial et de la période proto-urbaine, période pour laquelle il convient de parler de formation de la polis plutôt que de naissance, et ce dans des régions différentes, comme la Crète, la Laconie, et l'Italie. La première région est étudiée par Daniela Lefèvre-Novaro ${ }^{7}$, qui souligne que le phénomène de l'émergence de la polis, bien qu'il présente des éléments communs dans tout le monde grec, se développe de façon variée selon les contextes régionaux et locaux. Dans l'île de Crète, les recherches archéologiques de ces dernières décennies ont permis de progresser de manière significative dans l'étude du proto-urbanisme. En effet, il s'agit d'un processus évolutif, au cours duquel deux phases se distinguent, à savoir, d'après les mots de Daniela Lefèvre-Novaro, la « phase de formation » et la « phase de consolidation » de la cité-État.

9 L'âge du fer et les origines de Sparte constituent le sujet de l'article de Valeria Tosti ${ }^{8}$. L'abandon des sites de la Laconie, à l'âge du bronze tardif, correspondit au début d'une occupation intentionnelle de la future vallée de Sparte, site qui était presque inhabité précédemment. À l'époque protogéométrique et géométrique, il est possible d'entrevoir une articulation de l'espace qui est décrit par Thucydide (I, 10,2) comme kata kômas, c'est-à-dire par villages, et qui est plus génériquement le premier mode d'occupation des poleis grecques. La formation de la polis de Sparte s'inscrit dans un contexte de crise et de changements importants, qui concernent non seulement la Laconie mais plus généralement la Grèce tout entière. Après la crise et la chute des palais mycéniens, le vide administratif et même démographique fut comblé par un peuple venant probablement du nord, qui se mélangea progressivement aux populations autochtones. Ainsi s'expliquent aussi bien la discontinuité d'occupation spatiale que la discontinuité linguistique.

10 La période proto-urbaine est traitée également par Francesco Quondam ${ }^{9}$, même si la zone géographique est cette fois-ci l'Italie: c'est la phase pendant laquelle, durant quelques siècles autour de l'année 1000 avant notre ère, les communautés de villages disparurent pour laisser place dans la péninsule à de nouvelles formes d'agrégation territoriale, sociale et politique, préalables à la cité-État. L'Étrurie méridionale représente la région où le changement à la fin $\mathrm{du}_{\mathrm{II}}^{\mathrm{e}}$ millénaire avant notre ère est le 
plus évident, avec quatre centres proto-urbains majeurs, c'est-à-dire Vulci, Tarquinia, Cerveteri, Véies, mais le processus proto-urbain est attesté dans plusieurs régions de l'Italie, aussi bien au sud qu'au nord de la péninsule.

11 Même si, d'après Mogens H. Hansen, Aristote réduisait la polis à une communauté de citoyens, sans prendre en compte sa dimension de centre urbain, toujours est-il que dans l'histoire de la réflexion grecque sur la cité, sa conception représente le pilier autour duquel se réunirent et se fixèrent d'un point de vue théorique les hérédités de la polis archaïque et classique, mais se posèrent également les prémisses du concept nouveau de cité qui caractérise l'époque hellénistique ${ }^{10}$. Pour Aristote, la cité était une réalité multiple ${ }^{11}$, même s'il reconnaissait que son unité était son bien suprême ${ }^{12}$, tandis que selon Platon, les particularismes devaient être effacés pour créer une cité qui constituât un seul organisme unitaire ${ }^{13}$. Cette vision de la polis comme "unité dynamique » serait déjà présente chez Homère ${ }^{14}$. Dès ses origines, la cité se situerait entre deux polarités, l'une réunissant la diversité destinée à évoluer et à se transformer, l'autre visant à l'unité et à l'immobilité afin d'éliminer tout danger de stasis.

Un autre aspect de la conception aristotélicienne de la cité, déjà en filigrane de l'épopée homérique, concernait sa collocation comme omphalos par rapport à la terre et à la mer, afin que la connexion avec tous les points de son territoire fût facilitée ${ }^{15}$. Le véritable banc d'essai était, pour Aristote, l'harmonisation des schémas topographiques de la tradition avec la révolution urbanistique représentée par la diffusion du plan orthogonal hippodamien. Ce plan décrivait un modèle de cité complètement différent par rapport à la tradition précédente, modèle qui fut théorisé au v viècle av. J.-C., même si l'on peut déjà en distinguer des traces dans les fondations coloniales du $\mathrm{VII}^{\mathrm{e}}$ siècle av. J.-C. ${ }^{16}$, où était bien présente l'idée, qui accompagnait la fondation, d'une répartition rationnelle de l'espace selon un schéma prédéterminé, fondé sur les rues rectilignes, la forme quadrangulaire et la disposition régulière des îlots, à l'opposé de la cité " en forme de rue ", où le plan était constitué du cercle, de la ligne courbe et de la disposition irrégulière. C'était une véritable quadrature du cercle qu'Aristote entendait réaliser $^{17}$. Dans le livre VII de la Politique ${ }^{18}$, le plan hippodamien correspondrait à la cité idéale aristotélicienne ; néanmoins, celui-ci ne devait être appliqué qu'à une partie de la cité, probablement la zone basse, puisqu'il était propre à assurer la disposition agréable aux maisons privées, tandis que le schéma urbanistique ancien permettant une défense plus efficace devait être réservé à l'acropole. Cette vision urbanistique, où l'ancien plan est contaminé par le nouveau, mais les deux sont appliqués dans des zones distinctes de la cité, interpréterait également le régime idéal envisagé par Aristote, c'est-à-dire celui de la constitution mixte ${ }^{19}$ : l'oligarchie serait représentée par l'acropole organisée selon le plan ancien, tandis que la démocratie se refléterait dans la zone basse de la cité, organisée selon le plan hippodamien ${ }^{20}$. Une fondation qui était en accord avec ce modèle fut celle d'Alexandrie : la cité a été à bon titre définie «in bilico tra passato e futuro ${ }^{21}$ ", puisque son plan présentait aussi bien la forme régulière et quadrangulaire que la nature isthmique dotée de deux ports, dont le paradigme était la cité homérique des Phéaciens.

Une autre pierre angulaire du projet urbanistique de la polis idéale aristotélicienne était la création de deux agorai: l'une, l'agora haute, qui devait être tenue libre de marchandises et placée au-dessous des temples et des palais des magistrats, l'autre, l'agora basse, située aux marges de la cité et destinée au commerce. Cette idée 
aristotélicienne anticipait la diversification entre l'agora et l'agora commerciale qui se retrouva dans le plan urbanistique des cités grecques de l'époque hellénistique. Dans l'espace de l'agora « libre », réservé à l'oisiveté, se réalisait l'idée aristotélicienne de la cité comme lieu destiné non seulement à « vivre » mais à « bien vivre »².

L'introduction du plan orthogonal en Italie ne doit toutefois pas être entièrement attribuée à l'influence grecque. Les traces d'une cité étrusque, Misano ou Misa (en étrusque: "Kainua») près de Marzabotto, dans le nord de l'Italie (province de Bologne), sont le témoignage le plus important de l'utilisation par les Étrusques de plans orthogonaux ${ }^{23}$. Ce site ne serait pas antérieur au début du vie siècle av. J.-C. Le plan est assez clair : un cardo principal, avec une orientation nord-sud, qui était croisé, selon un schéma orthogonal, par trois decumani ; les lots étaient longs de 130 à $160 \mathrm{~m}$ et de largeur variable; il reste des attestations de pratiques cérémonielles lors de l'aménagement de Marzabotto, même si elles sont isolées. En effet, un cippe, pierre de fondation marquant le centre de la cité, gravée d'une croix, dont les bras suivaient les axes nord-sud et est-ouest a été mise au jour par les archéologues et confirmerait de fait les descriptions de Tite-Live (I, 17-18) concernant les rites de fondation des cités étrusques ${ }^{24}$. Dans ce volume, l'organisation spatiale de la civilisation étrusque entre les $\mathrm{VI}^{\mathrm{e}}$ et $\mathrm{IV}^{\mathrm{e}}$ siècles fait l'objet de l'article de Claire Joncheray ${ }^{25}$, avec entre autres l'exemple de Marzabotto: si la forme urbaine étrusque est assez bien documentée, l'usage politique de ces villes reste bien moins connu. Cependant, les centres urbains n'étaient pas le lieu exclusif du pouvoir dans un monde de migration et d'installations sur plusieurs terres.

Comme le présent ouvrage contribue à le mettre en relief, le phénomène de l'urbanisation concerna l'ensemble du bassin méditerranéen, occidental et oriental, de l'Italie à l'Europe celtique, à l'Asie Mineure; non seulement toutes les régions furent touchées, mais ce phénomène perdura tout au long de l'histoire ancienne dans la mesure où les cités furent fondées et refondées, des vagues migratoires furent à l'origine des installations coloniales grecques et les campagnes militaires et l'exigence de contrôler les nouveaux territoires à la base de la plupart des colonies romaines ${ }^{26}$. Les raisons pour constituer de nouvelles communautés sont différentes, d'après les phases qui se succédèrent dans l'histoire ancienne ${ }^{27}$.

16 L'expérience coloniale grecque est représentée par le cas particulier de Béziers I/Rhode, analysé par Élian Gomez et Daniela Ugolini ${ }^{28}$ : au début peut-être un simple emporion, le site devint assez vite une colonie (apoikia); les relations avec les indigènes se réorganisèrent au fil du temps mais finirent par se dégrader, notamment après la création rhodo-biterroise du port d'Agde I/Agathe, à la toute fin du vie siècle. Le cadre géographique de l'urbanisation est complété par l'article d'Andrea Fochesato sur l'Europe celtique ${ }^{29}$, qui, à partir de données archéologiques en constante augmentation ces dernières décennies, réexamine la question de l'apparition des oppida, agglomérations fortifiées dont le caractère urbain a été reconnu grâce aux fouilles de sites de référence comme l'oppidum de Bibracte, en Bourgogne. Ce phénomène urbain était déjà amorcé au $\mathrm{III}^{\mathrm{e}}$ siècle av. J.-C.

Dans un itinéraire géographique, de l'Ouest à l'Est, et chronologique, de l'âge du fer à l'époque hellénistique, les deux dernières études de la première partie traitent respectivement des villes de la Locride occidentale depuis l'âge du fer, notamment Naupacte, et de celles de la Carie à l'époque hellénistique. L'article de Despina Chatzivasiliou $^{30}$ porte sur la structuration par kômai de la Locride de l'Ouest, parmi 
lesquelles Naupacte, située à l'extrémité ouest de la région: malgré la pénurie de vestiges archéologiques, il est possible d'entrevoir les étapes de la formation des sites en prenant en considération les sources littéraires et l'organisation de l'ensemble du territoire. En revanche, le paysage urbain de la Carie hellénistique fait l'objet de l'article de Gabrièle Larguinat-Turbatte ${ }^{31}$ : dans la région, à cette époque, le phénomène d'urbanisation de type grec serait étroitement lié à l'adoption du modèle politique et culturel de la cité grecque.

La deuxième partie du volume est consacrée aux questions de terminologie à travers quatre articles, qui concernent le monde romain occidental, de l'espace ibérique à l'Afrique du Nord, sur un arc chronologique assez large, de l'époque de César à l'Antiquité tardive. L'approche diachronique permet de cerner les modifications du rôle et $\mathrm{du}$ fonctionnement de la cité et l'évolution linguistique qui accompagna les changements se produisant sur plusieurs siècles.

Le premier des articles de cette section est de Sabine Lefebvre ${ }^{32}$, et porte sur le terme oppidum dans une œuvre postérieure à la guerre des Gaules, à savoir le Bellum ciuile de César, ce qui amène d'une part à s'interroger sur le choix du terme oppidum par César, et d'autre part à proposer des traductions différentes du mot: après la guerre des Gaules, l'oppidum fait référence pour César à des sites particuliers, comme Bibracte ou Vesontio; en revanche, son usage dans le Bellum ciuile témoigne d'une acception plus large, en vertu de laquelle, pour César, l'oppidum serait un noyau urbain assez conséquent, une ville.

21 Le deuxième article, de Susanna Marcos ${ }^{33}$, porte sur les aspects juridiques et théoriques de la ciuitas dans un contexte de réorganisation des communautés des provinces au Haut-Empire, notamment en Lusitanie : à travers l'étude du vocabulaire, l'article se focalise tour à tour sur l'imbrication du populus, de l'oppidum et de la ciuitas, en envisageant également la hiérarchisation juridico-administrative urbaine de l'Empire et les conceptions politiques qui leur étaient intimement liées, et, parfois, la réalité de la pratique quotidienne.

Dans le troisième article, Anne-Florence Baroni ${ }^{34}$ revient sur la vexata quaestio du terme castellum: ce mot apparaît fréquemment dans la documentation des provinces africaines pour désigner une agglomération rurale, toutefois sa définition précise n'est pas clairement établie ; l'article, remettant en cause les explications les plus courantes dans la bibliographie, qui mettent l'accent sur la question de la fortification du castellum, de son rapport avec le pagus, et de son statut pérégrin, propose une définition assez simple du castellum comme agglomération secondaire sans statut particulier, définition qui aurait le mérite d'embrasser les différents cas de figure, comme les castella de la Confédération cirtéenne, les castella gérés par des magistrats pérégrins du territoire de Sicca Veneria, le castellum Victoriae sur le territoire d'Igilgili, mais aussi les castella maurétaniens regroupant des colons des domaines impériaux.

Le dossier se termine par l'article de Christian Stein ${ }^{35}$ qui revient sur les termes ciuitas et urbs dans un espace très périphérique de l'Empire romain $\mathrm{du} \mathrm{v}^{\mathrm{e}}$ siècle, étudié à travers la Chronique d'un auteur occidental tardif, Hydace: le monde s'y réduit pour l'essentiel à l'espace ibérique dans le cadre plus large d'une géographie de plus en plus chrétienne; ciuitas et urbs semblent y être interchangeables, mais certains indices pourraient indiquer un rôle plus important du premier terme; enfin, ce qui fait l'importance d'une ville chez Hydace n'a rien à voir avec sa dimension proprement 
urbaine, mais dépend presque entièrement de l'aura de son siège épiscopal ou de l'aura personnelle remarquable de ses évêques.

Les dernières études de ce recueil interviennent sur certains aspects du phénomène qui est connu sous le nom de «romanisation ${ }^{36}$ " et qui a fortement contribué à l'urbanisation de régions où le nombre de centres urbains n'était pas précédemment significatif. Le sujet des fondations est par ailleurs étroitement lié à un autre thème, à savoir celui de la destruction de cités. Comme la fondation d'une ville était sanctionnée par des rites religieux, il n'en allait pas différemment pour sa destruction, surtout si elle intervenait à la suite d'une défaite militaire. Dans le cadre romain, il s'agissait du rite de l'euocatio, c'est-à-dire l'acte de procédure religieuse par lequel les dieux d'une cité ennemie étaient invités, au moment de l'assaut final, à « abandonner le territoire, les temples, les cultes et la ville» de l'ennemi, pour accorder leur préférence "au territoire, aux temples, aux cultes et à la ville » de Rome ${ }^{37}$. Autrement dit, la cité, dans toutes les phases de son histoire et dans son organisation spatiale, reflétait en profondeur les aspects politiques, religieux, militaires, du monde gréco-romain, aussi bien du point de vue des élaborations théoriques dont elle était le point de départ essentiel, que de la vie quotidienne et de la répartition de l'espace. Par conséquent, l'évolution de la cité antique et ses transformations urbanistiques restent des sujets fondamentaux pour comprendre les structures sociale et politique ainsi que les phénomènes migratoires et leurs retombées dans tout le bassin de la Méditerranée antique. C'est aussi pour cette raison que la réflexion autour de l'organisation de la cité rejoignit assez précocement celle sur la communauté idéale et sur le meilleur régime politique $^{38}$.

Par ailleurs, la spéculation autour de la cité idéale n'ignorait pas la réalité dystopique des villes antiques, parmi lesquelles Rome elle-même ${ }^{39}:$ "To indulge in any exercise that asserts that life in Ancient Rome was 'good' or 'bad' is to pursue a rhetorical exercise familiar to the ancients ${ }^{40} . »$

\section{BIBLIOGRAPHIE}

CARSANA Chiara, La teoria delta «costituzione mista» nell'età impériale romana, Biblioteca di Athenaeum, 13, Côme, Edizioni New Press, 1990.

CASTAGNOLI Ferdinando, Orthogonal Town Planning in Antiquity, Cambridge (MA), MIT Press, 1971.

CLARK Peter, The Oxford Handbook of Cities in World History, Oxford, OUP, 2013.

COUDRY Marianne \& SCHETTINO Maria Teresa (éd.), L'utopie politique et la cité idéale, dossier thématique de la revue Politica antica, 5, 2015.

COUDRY Marianne \& SCHETTINO Maria Teresa (éd.), Enjeux interculturels de l'utopie politique dans l'Antiquité gréco-romaine, Alexandrie, Edizioni dell'Orso, sous presse.

GRECO Emanuele, La città greca antica. Istituzioni, società e forme urbane, Rome, Donzelli, 1999. 
HANSEN Mogens H. (éd.), Acts of the Copenhagen Polis Centre 1-7, Copenhague, The Royal Danish Academy of Sciences and Letters, 1993-2005.

HANSEN Mogens H. (éd.), Papers from the Copenhagen Polis Centre 1-8 = Historia, Einzelschriften 87, $95,108,117,138,162,180,198$, Stuttgart, Steiner, 1994-2007.

HANSEN Mogens H., Polis and City-State. An Ancient Concept and Its Modern Equivalent (Symposium, 9 January 1998, Acts of the Copenhagen Polis Centre, vol. 5), Copenhague, The Royal Danish Academy of Sciences and Letters, 1998 (Polis et cité-État, trad. française, Paris, 2001).

HANSEN Mogens H. (éd.), A Comparative Study of Thirty City-State Cultures, Copenhague, The Royal Danish Academy of Sciences and Letters, 2000.

HANSEN Mogens H. (éd.), A Comparative Study of Six City-State Cultures, Copenhague, The Royal Danish Academy of Sciences and Letters, 2002.

HANSEN Mogens H., Polis: An Introduction to the Ancient Greek City-State, Oxford, OUP, 2006 (Polis. Une introduction à la cité grecque, trad. française, Paris 2008).

HANSEN Mogens H. \& FISCHER HANSEN Tobias, « Monumental Political Architecture in Archaic and Classical Greece », dans D. Whitehead (éd.), From Political Architecture to Stephanus Byzantius: Sources for the Ancient Greek Polis, Historia, Einzelschriften 87, Stuttgart, Steiner, 1994, p. 23-90.

HANSEN Mogens H. \& RAAFLAUB Kurt (éd.), Studies in the Ancient Greek Polis, Historia, Einzelschriften 95, Stuttgart, Steiner, 1995.

HANSEN Mogens H. \& NIELSEN Thomas H. (éd.), Inventory of Archaic and Classical Poleis, Oxford, OUP, 2004.

LAURENCE Ray, « Writing the Roman Metropolis », dans H. M. Parkins (éd.), Roman Urbanism. Beyond the Consumer City, Londres/New York, Routledge, 1997, p. 1-20.

LOMAS Kathryn, « The Idea of a City: Élite Ideology and the Evolution of Urban Form in Italy, 200 BC-AD 100 », dans H. M. Parkins (éd.), Roman Urbanism. Beyond the Consumer City, Londres/ New York, Routledge, 1997, p. 21-41.

MANSUELLI Guido A., « Marzabotto. Dix années de fouilles et de recherches », MEFR, 84, 1972, p. 111-144.

MORLEY Neville, « Cities in Context: Urban Systems in Roman Italy », dans H. M. Parkins (éd.), Roman Urbanism. Beyond the Consumer City, Londres/New York, Routledge, 1997, p. 42-58.

MUSTI Domenico, Lo scudo di Achille. Idee e forme di città nel mondo antico, Rome/Bari, Laterza, 2008. PARKINS Helen M., «The 'Consumer City’ Domesticated? The Roman City in Élite Economic Strategies », dans H. M. Parkins (éd.), Roman Urbanism. Beyond the Consumer City, Londres/ New York, Routledge, 1997, p. 83-111

RYKWERT Joseph, The Idea of a Town. The Anthropology of Urban Form in Rom, Italy and the Ancient World, Londres, 1976.

SANTUCCI Marco, « Forme dello spazio e forme della politica nella città aristotelica », dans P. A. Bernardini (éd.), La città greca. Gli spazi condivisi, Pise/Rome, Fabrizio Serra Editore, 2014, p. $107-125$.

SCHULLER Wolfgang, HOEPFNER Wolfram \& SWANDER Ernst L. (éd.), Demokratie und Architektur. Des hippodamische Städteban und die Entstehung der Demokratie, Munich, Deutscher Kunstverlag, 1989. 


\section{NOTES}

1. A. Esposito appartient à l'UMR 6298 ARTEHIS (Université de Bourgogne, CNRS) ; A. Pollini à

l'UMR 7044 ARCHIMÈDE (Université de Strasbourg, Université de Haute-Alsace, CNRS).

2. Hansen \& Nielsen (2004).

3. Voir Hansen (1993-2005), Hansen \& Raaflaub (1995) ; cf. également infra, la note suivante.

4. Hansen (1998, trad. française 2001) ; cf. aussi Hansen (2006, trad. française 2008), Hansen (1994-2007).

5. Hansen (2000) ; cf. aussi Hansen (2002) et sur le même sujet Clark (2013).

6. Cf. en particulier Hansen \& Fischer Hansen (1994, 23-90).

7. D. Lefèvre-Novaro, «Le processus de formation des poleis en Crète: état de la question et perspectives de recherche ».

8. Valeria Tosti, «La naissance de Sparte : entre sources littéraires et sources archéologiques ».

9. Francesco Quondam, «L'Italia dei centri proto-urbani: percorsi regionali a confronto ».

10. Cf. Santucci $(2014,107-125)$.

11. Aristote, La Politique, II, 1261a, 18.

12. Aristote, La Politique, II, $1261 \mathrm{a}, 15$

13. Platon, La République, V, 462a-d.

14. Voir Santucci $(2014,110)$.

15. Aristote, La Politique, VII, 1330b, 19-21.

16. Voir Castagnoli (1971).

17. Cf. le projet paradoxal décrit par l'architecte Méton dans Les oiseaux d'Aristophane, comédie représentée aux Lénéennes en 414 av. J.-C. (v. 1004-1009).

18. Aristote, La Politique, VII, 1330b, 17-26.

19. Voir Aristote, La Politique, VII, 1330a, 34-36: « une acropole sert à un régime oligarchique et monarchique, tandis qu'un lieu dans la plaine est plus propice à la démocratie »; cf. Aristote, La Politique, VII, 1330b, 7-12 et 1330b, 17-21. Sur la constitution mixte, voir Carsana (1990) ainsi que les travaux menés dans le cadre du programme scientifique coordonné par Stavroula Kefallonitis dans l'UMR 5189 HISOMA (Université Lumière Lyon 2, Université Lyon 3, Université de Saint-Étienne, ENS de Lyon, CNRS).

20. Voir Santucci $(2014,117)$. Sur la cité idéale et le lien entre le plan hippodamien et le régime démocratique, voir aussi Schuller, Hoepfner \& Swander (1989).

21. Musti $(2008,72)$.

22. Aristote, La Politique, I, 1252b, 29-30. Cf. aussi Santucci (2014, 121-122).

23. Voir Mansuelli (1972,111-144). Cf. aussi Rykwert (1976, 71-96).

24. Sur les cippi et la problématique du périmètre de la ville, voir Rykwert (1976, 97-162).

25. Claire Joncheray, «Vocabulaire de la cité et formes urbaines dans le monde étrusque ».

26. Sur le choix des sites où fonder de nouvelles villes, voir, entre autres, Rykwert (1976, 33-34 et 41-71). Cicéron liste les raisons géographiques, économiques et hygiéniques qui justifiaient le choix de Romulus ; voir Cicéron, De la République, II, 5, 10 : « [...] Qui potuit igitur diuinius et utilitates conplecti maritimas Romulus et uitia uitare, quam quod urbem perennis amnis et aequabilis et in mare late influentis posuit in ripa? quo posset urbs et accipere a mari quo egeret, et reddere quo redundaret, eodemque ut flumine res ad uictum cultumque maxime necessarias non solum mari absorberet, sed etiam inuectas acciperet ex terra, ut mihi iam tum diuinasse ille uideatur hanc urbem sedem aliquando et domum summo esse imperio praebituram; nam hanc rerum tantam potentiam non ferme facilius alia ulla in parte Italiae posita urbs tenere potuisset. " Sur l'importance des conditions climatiques, voir Vitruve, I, 6 , 7. Le site choisi pouvait déterminer le futur d'une ville et contribuer à la création de la cité

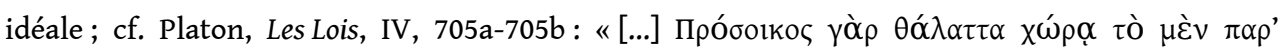

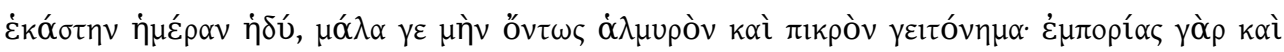

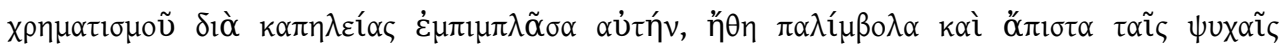




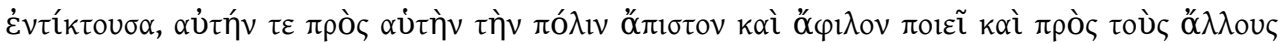

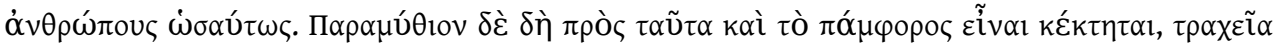

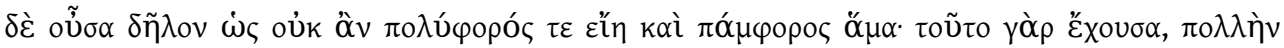

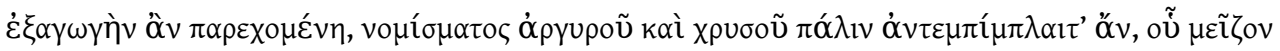

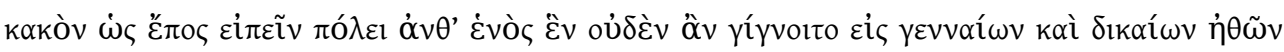

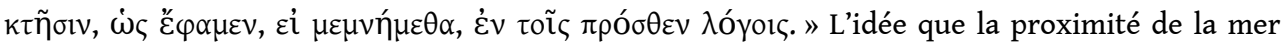
pouvait être un facteur de corruption des mœurs est également présente chez Cicéron, De la République, II, 4.

27. Voir Rykwert (1976, 30-31).

28. Élian Gomez et Daniela Ugolini, « Les premiers Grecs en France : le cas de Béziers I/Rhode ».

29. Andrea Fochesato, «L'emergenza del fenomeno urbano nell'Europa continentale della seconda età del ferro: Bibracte e gli oppida celtici ».

30. Despina Chatzivasiliou, «La Locride de l'Ouest et la première forme urbaine de la ville de Naupacte ».

31. Gabrièle Larguinat-Turbatte, «Constitution d'un paysage urbain et diffusion du modèle de la cité grecque en Carie à l'époque hellénistique ».

32. Sabine Lefebvre, «Traduire le terme oppidum : un exemple des difficultés posées par le Bellum ciuile de César ».

33. Susana Marcos, «Ce que disent les mots. Nommer la ville lusitanienne ».

34. Anne-Florence Baroni, «Le vocabulaire des établissements urbains antiques et les incertitudes du mot castellum en Afrique ».

35. Christian Stein, «La géographie humaine d'Hydace ».

36. La bibliographie sur le sujet est trop vaste et connue, aussi bien sur la romanisation en général que sur les aspects régionaux de ce phénomène historique, pour être citée ici. Sur le rôle joué par les élites dans le processus de romanisation notamment en Italie, ce qui comporta une transformation de la forme urbaine, voir Lomas (1997, 21-41). Sur le système urbain en Italie, voir également Morley (1997, 42-58). Sur le paradigme wébérien de la «cité consommatrice ", repris par Moses Finley, voir Parkins (1997, 83-111).

37. Le texte qui nous est parvenu concerne la prise de Carthage par Scipion Émilien en 146 av. J.-C.; il est transmis par Macrobe, Saturnales, III, 9, 7-12: «Est autem carmen huiusmodi quo di euocantur, cum oppugnatione ciuitas cingitur: 'Si deus si dea est cui populus civitasque Carthaginiensis est in tutela, teque maxime, ille qui urbis huius populique tutelam recepisti, precor venerorque veniamque a vobis peto ut vos populum civitatemque Carthaginiensem deseratis, loca templa sacra urbemque eorum relinquatis absque his abeatis, eique populo civitati metum formidinem oblivionem iniciatis, proditique Romam ad me meosque veniatis, nostraque vobis loca templa sacra urbs acceptior probatiorque sit, mihique populoque Romano militibusque meis praepositi sitis ut sciamus intelligamusque. Si ita feceritis, voveo vobis templa ludosque facturum'. In eadem uerba hostias fieri oportet auctoritatemque uideri extorum, ut ea promittant futura. Urbes uero exercitusque sic deuonentur iam numinibus euocatis, sed dictatores imperatoresque soli possunt deuouere his uerbis: 'Dis pater Veiovis Manes, sive quo alio nomine fas est nominare, ut omnes illam urbem Carthaginem exercitumque quem ego me sentio dicere fuga formidine terrore conpleatis, quique adversum legiones exercitumque nostrum arma telaque ferent, uti vos eum exercitum eos hostes eosque homines urbes agrosque eorum et qui in his locis regionibusque agris urbibusve habitant abducatis lumine supero privetis exercitumque hostium urbes agrosque eorum quos me, sentio dicere, uti vos eas urbes agrosque capita aetatesque eorum devotas consecratasque habeatis ollis legibus quibus quandoque sunt maxime hostes devoti. Eosque ego vicarios pro me fide magistratuque meo pro populo Romano exercitibus legionibusque nostris do devoveo, ut me meamque fidem imperiumque legiones exercitumque nostrum qui in his rebus gerundis sunt bene salvos siritis esse. Si haec ita faxitis ut ego sciam sentiam intellegamque, tunc quisquis hoc votum faxit ubi faxit recte factum esto ovibus atris tribus. tellus mater teque iuppiter obtestor'. Cum Tellurem dicit, manibus terram tangit : cum Iouem dicit, manus ad caelum tollit : cum uotum recipere dicit, manibus pectus tangit. » 
38. Voir sur ce sujet Coudry \& Schettino (2015) et Coudry \& Schettino (sous presse). Voir aussi Rykwert (1976).

39. Voir Laurence (1997, 1-20).

40. Laurence $(1997,18)$.

\section{AUTEUR}

\section{MARIA TERESA SCHETTINO}

Université de Haute-Alsace, Université de Strasbourg, UMR 7044 Archimède, CNRS

mtschettino@misha.fr 